\title{
Birthweight, Childhood Body Mass Index, Height and Growth, and Risk of Polycystic Ovary Syndrome
}

\author{
Julie Aarestrup ${ }^{a}$ Dorthe C. Pedersen ${ }^{a}$ Peter E. Thomas ${ }^{a} \quad$ Dorte Glintborg ${ }^{b}$ \\ Jens-Christian Holm ${ }^{c, d}$ Lise G. Bjerregaard ${ }^{a}$ Jennifer L. Baker ${ }^{a, d}$ \\ ${ }^{a}$ Center for Clinical Research and Prevention, Bispebjerg and Frederiksberg Hospital, The Capital Region, \\ Frederiksberg, Denmark; ${ }^{b}$ Department of Endocrinology and Metabolism, Odense University Hospital, University \\ of Southern Denmark, Odense, Denmark; 'The Children's Obesity Clinic, European Center of Management (EASO), \\ Department of Pediatrics, Holbæk University Hospital, Holbæk, Denmark; ${ }^{d}$ Novo Nordisk Foundation Center for \\ Basic Metabolic Research, Human Genomics and Metagenomics in Metabolism, Faculty of Health and Medical \\ Sciences, University of Copenhagen, Copenhagen, Denmark
}

\section{Keywords}

Birthweight · Body height · Body weights and measures ·

Child · Polycystic ovary syndrome

\begin{abstract}
Introduction: Adult obesity is linked with polycystic ovary syndrome (PCOS), but the importance of body size at ages before PCOS is diagnosed is unknown. Objective: To investigate associations between a woman's own birthweight, childhood body mass index (BMI), height and growth patterns in relation to her risk of PCOS. Methods: We included 65,665 girls from the Copenhagen School Health Records Register, born in the period 1960-1996, with information on birthweight and measured weight and height at the ages of 7-13 years. Overweight was defined using International Obesity Task Force (IOTF) criteria. From the Danish National Patient Register, 606 women aged $15-50$ years were identified. Hazard ratios (HRs) and 95\% confidence intervals (Cls) were estimated by Cox regression analysis. Results: Birthweight was not associated with PCOS. At the age of 7-13 years, girls with overweight had a higher risk of developing PCOS than girls without overweight; HR $2.83(95 \% \mathrm{Cl} 2.34$ 3.42) at age 7 years and $2.99(95 \% \mathrm{Cl} 2.38-3.76)$ at age 13
\end{abstract}

karger@karger.com www.karger.com/ofa

Karger $\stackrel{\text { ' }}{5}$

GOPEN ACCESS
(C) 2021 The Author(s)

Published by S. Karger AG, Basel

This is an Open Access article licensed under the Creative Commons Attribution-NonCommercial-4.0 International License (CC BY-NC) (http://www.karger.com/Services/OpenAccessLicense), applicable to the online version of the article only. Usage and distribution for commercial purposes requires written permission. years. Furthermore, girls with overweight at both 7 and 13 years had a higher risk of developing PCOS than girls without overweight or overweight at only one age. Height was positively associated with PCOS risk at all ages. Girls who were persistently tall or changed from tall to average height had a higher risk of developing PCOS than girls with average height growth. Conclusion: Overweight and tall stature in childhood are positively associated with PCOS risk, but birthweight is not.

(C) 2021 The Author(s) Published by S. Karger AG, Basel

\section{Introduction}

Polycystic ovary syndrome (PCOS) is one of the most common endocrine conditions to affect approximately $5-13 \%$ of women of reproductive age, depending on the source population and diagnostic criteria [1-3]. The disorder is characterized by hyperandrogenism, oligoanovulation, and polycystic ovaries. PCOS often emerges during adolescence and affects a woman throughout her entire life. PCOS negatively impacts quality of life and is associated with reproductive and metabolic complications as well as numerous chronic diseases including type 
2 diabetes, metabolic syndrome, cardiovascular disease, psychiatric disorders, and cancer at several sites [2, 4-6].

PCOS is a complex disorder with a heterogenous clinical presentation and a poorly understood multifactorial etiology $[1,2]$. As such, there are very few well-established risk factors. PCOS and overweight are closely interrelated, and an estimated $50-70 \%$ of women with PCOS are affected by overweight $[7,8]$. Furthermore, obesity may exacerbate adverse health conditions in women with PCOS [1]. As clinical manifestations of PCOS often emerge during the teenage years, the preceding years from birth and throughout childhood may be a critical period for this condition to develop. Birthweight, as an indicator of the intrauterine environment, may be inversely associated with PCOS risk but there is uncertainty about this, as the association is not significant in largescale studies, suggesting a publication bias [9]. Six studies have investigated links between body size in childhood and a subsequent risk of developing PCOS, and generally found positive associations [10-15]. However, these studies were limited by using self-reported diagnoses of PCOS or its symptoms only, and most studies assessed childhood body size by self-report. Interestingly, height is consistently and inversely associated with the risk of developing cardiometabolic disease [16], but no studies have evaluated childhood height in relation to PCOS risk.

Taken together, the current evidence regarding a link between early-life body size and a subsequent risk of developing PCOS is suggestive but inadequate. The aim of this study was to examine, in a large Danish populationbased cohort, if birthweight, childhood body mass index (BMI) and height at the ages of 7-13 years, change in weight status, and growth in height during this period are associated with the risk of developing PCOS.

\section{Materials and Methods}

\section{Cohort}

The study was based on the Copenhagen School Health Records Register (CSHRR) [17], which currently contains digitalized information on 406,350 children (200,977 girls). Virtually all children born in the period 1930-1996 who attended a public or private school in the municipality of Copenhagen, Denmark, are included in this register. During their school years (i.e., 7-13 years), the children underwent health examinations performed by trained school physicians or nurses; these included the measurement of height (without shoes) and weight (naked or in underwear only). Birthweight was reported by the parent or caregiver at the first school health examination (at approx. 7 years of age), either from recall or from a medical birth record, and is available for children born in 1936 onwards. Correlations between birthweight values from the CSHRR and electronic records are high (0.93-0.97) [18].
Also included in the CSHRR are unique government-issued identification (ID) numbers for the children. Since 1968, all Danish citizens have been issued these numbers, which then can be used to perform individual-level linkages with national registries [19]. The ID number was recorded on the health cards for children attending school at this time or later and could be retrieved for those who left school before 1968 [17].

\section{Linkages and Outcomes}

PCOS diagnoses were identified from discharge diagnoses obtained via linkage to the Danish National Patient Register. This register contains all inpatient (since 1977) and outpatient (since 1995) hospital contacts. We identified diagnoses of PCOS according to the International Classification of Disease (ICD), the 8th revision of which was used in Denmark until 1994 and the 10th revision thereafter. The first hospital admission with the following codes was used to define PCOS: ICD-8: 256.9 and ICD-10: E28.2. A diagnosis of PCOS should only be established after ruling out disorders that may cause similar symptoms [20]. We identified the following relevant disorders: hypofunction or hyperfunction of the pituitary gland including hyperprolactinemia and acromegaly (ICD-8: 253.0; ICD-10: E22.0 and E22.1), Cushing's disease (ICD8: 258.0; ICD-10: E24.0), adrenogenital syndrome (ICD-8: 255.0255.1; ICD-10: E25.0), and Turner syndrome (ICD-8: 759.5; ICD10: Q96.0). Through the ID number, individual-level linkage of girls in the CSHRR to the Danish Civil Registration System for vital status information was performed [19].

\section{Study Population}

Women eligible for this study were born in 1960-1996, had an ID number, were still alive and living in Denmark on 1 January 1977 or later, and were aged $\geq 15$ years. Among these 68,357 women, those with one of the disorders listed above or who had undergone bilateral oophorectomy $(n=35)$ before the age of 15 years were excluded (online suppl. Fig. S1; for all online suppl. material, see www.karger.com/doi/10.1159/000515294). Women were also excluded if they had been diagnosed with PCOS before the age of 15 years $(n=11)$, to avoid the diagnosis potentially occurring before the childhood exposures and because PCOS is usually difficult to diagnose at these ages. Women with childhood height and BMI $Z$ scores that were missing $(n=2,643)$ or outlying ( $Z$ score $<-4.5$ or $>4.5 ; n=3$ ) at all childhood ages were excluded. This resulted in an analytical cohort of 65,665 women.

For the analysis of the association between birthweight and PCOS, women with missing birthweight information $(n=9,611)$ or with a birthweight value outside the reliable range of $2.0-5.5 \mathrm{~kg}$ ( $n=754)$ were excluded, resulting in 55,300 women being available for the analysis.

We followed the girls from 1 January 1977, or the age of 15 years (whichever came later) until they: reached the age of 50 years (to avoid inclusion of a postmenopausal PCOS diagnosis as this is rare and likely represents another etiology); received a diagnosis of PCOS, one of the PCOS-related disorders listed above, or bilateral oophorectomy; died; emigrated; were lost to follow-up; or until 30 June 2017 (whichever came first).

\section{Statistical Analyses}

Childhood BMI and height values were transformed into agespecific $Z$ scores (for the ages 7-13 years) using an internal reference and the lambda mu sigma (LMS) method [21]. Using Inter- 
Table 1. Early-life body size characteristics of the study population of girls from the Copenhagen School Health Records Register

\begin{tabular}{lccccc}
\hline Characteristic & Age, years & Number & Median & IQR & $\begin{array}{l}\text { Proportion with } \\
\left.\text { overweight }{ }^{1}, \mathrm{~kg} / \mathrm{m}^{2}\right)\end{array}$ \\
\hline Birthweight, kg & Birth & 55,300 & 3.30 & $3.00-3.60$ & \\
\hline BMI & 7 & 59,878 & 15.5 & $14.6-16.6$ & $11.4(\geq 17.69)$ \\
& 8 & 58,475 & 15.8 & $14.8-17.0$ & $11.8(\geq 18.28)$ \\
& 9 & 51,080 & 16.2 & $15.1-17.6$ & $12.7(\geq 18.99)$ \\
& 10 & 46,587 & 16.6 & $15.4-18.3$ & $12.9(\geq 19.78)$ \\
& 11 & 45,668 & 17.1 & $15.8-18.9$ & $12.4(\geq 20.66)$ \\
& 12 & 45,021 & 17.8 & $16.3-19.7$ & $12.5(\geq 21.59)$ \\
Height, cm & 13 & 42,340 & 18.7 & $17.1-20.6$ & \\
& 7 & 59,878 & 123.2 & $119.9-126.8$ & \\
& 8 & 58,475 & 128.7 & $125.1-132.4$ & \\
& 9 & 51,080 & 134.2 & $130.3-138.1$ & \\
& 10 & 46,587 & 139.6 & $135.5-144.0$ & \\
& 11 & 45,668 & 145.7 & $141.0-150.5$ & \\
\hline
\end{tabular}

IQR, interquartile range; BMI, body mass index.

${ }^{1}$ Overweight as defined by the International Obesity Task Force criteria.

national Obesity Task Force (IOTF) criteria, BMI values were classified as non-overweight or overweight (including obesity) [22]. Characteristics of the cohort are presented as median and interquartile range (IQR) at birth and at the ages 7-13 years as well as the proportion of girls with overweight.

Cox proportional hazard regression was used to investigate the associations between birthweight, childhood BMI, and height $Z$ scores, change in childhood weight status, and growth in height, respectively, and the risk of developing PCOS. The estimates are presented as hazard ratios (HRs) with $95 \%$ confidence intervals (CIs). Age was used as the underlying time axis and all analyses were Cox-stratified by birth cohort (1960-1964, 1965-1969, ..., 1990-1996) to allow the baseline hazards to differ by birth cohort.

The shape of the associations between birthweight and PCOS was evaluated using a linear spline model with 2 knot points positioned at 3.25 and $3.75 \mathrm{~kg}$ and then tested against a linear model using the likelihood ratio test. We did not detect deviations from linearity $(p=0.14)$. Nonetheless, associations are presented both linearly (per kilogram) and in 5 categories (2.00-2.75, 2.76-3.25, $3.26-3.75,3.76-4.25$, and $4.26-5.50 \mathrm{~kg}$ ). These categories were selected to reduce the impact of digit preference [23]. For childhood BMI and height, respectively, we investigated the shapes of the associations using piecewise linear splines with 3 knots positioned at $Z$ scores of $-0.68,0$, and +0.68 (corresp. approx. to the 25 th, 50 th, and 75 th percentiles) in comparison with a linear model using the likelihood ratio test. For the BMI and PCOS associations, there were deviations from linearity $(p \leq 0.02)$. The model with a single knot at zero most appropriately represented the shape of the associations ( $p \leq 0.0029)$; associations are thus presented per BMI $Z$ score in the girls with a $Z$ score of $\geq 0$ or $<0$, respectively. We did not detect any deviations from linearity in the associations between childhood height and PCOS risk ( $p \geq 0.25)$, so height was modeled linearly per $Z$ score. We also estimated PCOS risk in girls with overweight in comparison with girls without overweight.

In girls with information on body size at the age of 7 and 13 years, we investigated whether change in weight status and growth in height, respectively, across these ages, were related to the risk of developing PCOS. Girls who remained in the non-overweight category between 7 and 13 years, or who changed to a higher or lower BMI category, were compared with the reference group of girls who stayed in the overweight category. In a second model, we defined girls without overweight at both ages as the reference group. Growth in height was investigated using a model with height at the age of 7 and 13 years as continuous variables and another model with height at age 7 years and change in height between the ages of 7 and 13 years as continuous variables. From these models, hypothetical growth patterns were derived and related to the risk of developing PCOS. Since few girls change by $> \pm 0.5$ height $Z$ scores between the ages of 7 and 13 years, an above-average change was defined as a change of $0.5 Z$ score and a below-average change as a $-0.5 Z$ score.

We investigated the assumption of proportional hazards by testing if the associations between early-life body size and the risk of PCOS differed by categories of age at risk using the likelihood ratio test. No violations of the proportional-hazards assumption were detected $(p \geq 0.06)$. In addition to using a Cox stratification for birth cohort in all analyses, which allows the baseline hazard to differ, we further formally examined if there were interactions by birth cohort on the associations between early-life body size and the risk of PCOS using the likelihood ratio test. No birth cohort interactions were observed (all $p$ values were $\geq 0.18$, except for one $p$ value of 0.04 for BMI at 12 years). Finally, we investigated a potential interaction between birthweight and childhood BMI in the associations with PCOS risk using the likelihood ratio test but did 
Table 2. Associations between birthweight and the risk of polycystic ovary syndrome ${ }^{1}$

\begin{tabular}{lrrll}
\hline & $N$ & $n$,cases HR & $95 \% \mathrm{CI}$ \\
\hline Birthweight (per kg) & 55,300 & 503 & 0.92 & $0.77-1.10$ \\
Birthweight category & & & \multicolumn{3}{c}{$0.68-1.20$} \\
$2.00-2.75 \mathrm{~kg}$ & 7,419 & 64 & 0.90 & $0.82-1.23$ \\
$2.76-3.25 \mathrm{~kg}$ & 18,205 & 176 & 1.00 & 0.00 (ref.) \\
$3.26-3.75 \mathrm{~kg}$ & 19,905 & 192 & 1.00 \\
$3.76-4.25 \mathrm{~kg}$ & 8,019 & 56 & 0.73 & $0.54-0.98$ \\
$4.26-5.50 \mathrm{~kg}$ & 1,752 & 15 & 0.90 & $0.53-1.52$ \\
\hline
\end{tabular}

$\mathrm{HR}$, hazard ratio; CI, confidence interval.

${ }^{1}$ Stratified by birth cohort.

not detect any (all $p$ values $\geq 0.06$, except for one $p$ value of 0.03 for BMI at 13 years). The statistical analyses were conducted using Stata v15.1.

\section{Results}

The study population consisted of 65,665 women with at least 1 measurement of childhood weight and height. During 1.5 million person-years of follow-up and a median of 23.6 years of observation time per person, 606 women were diagnosed as having PCOS between the ages of 15 and 50 years, with a median age at diagnosis of 28 years (IQR 23-32 years). The women were diagnosed with PCOS in the period 1979-2017, with approximately $90 \%$ of these diagnoses being made from the early 2000 s onwards. Median birthweight was $3.3 \mathrm{~kg}$. During childhood, in all girls, BMI and height increased with age, as expected (Table 1 ).

Of the 55,300 girls with available information on birthweight, 503 women were diagnosed with PCOS during follow-up. Birthweight was not associated with PCOS risk in the linear analyses (Table 2). Furthermore, we did not find consistent associations between birthweight and PCOS risk in the categorical analyses, albeit girls with birthweights of $3.76-4.25 \mathrm{~kg}$ had a reduced risk of PCOS compared to those with birthweights of $3.26-3.75 \mathrm{~kg}$ (Table 2).

Across all childhood ages, girls with an above-average BMI ( $Z$ score $\geq 0)$ had an increased risk of PCOS (Fig. 1; online suppl. Table S1). At the age of 7 years, the HR for PCOS was 1.73 (95\% CI 1.57-1.91) per BMI $Z$ score among girls with an above-average BMI. In contrast, no associations between BMI and risk of PCOS were found in girls with a below-average $\mathrm{BMI}(Z$ score $<0)$. At the age of 7 years, the HR for PCOS was 1.01 (95\% CI 0.83-1.23) per BMI $Z$ score among girls with a $Z$ score $<0$. The estimates were similar at the other childhood ages. When categorizing BMI values as non-overweight or overweight, girls with overweight had an approximately 3 -fold higher risk of PCOS than girls without overweight at all childhood ages (Table 3 ).

In the 38,285 girls with available childhood body size information at both ages, i.e., 7 and 13 years, 295 were diagnosed with PCOS during follow-up. Compared to girls with overweight at the age of 7 and 13 years, those without overweight at both ages and those with overweight at only one age had a lower risk of PCOS (Table 4). When girls without overweight at both ages were used as the reference group, those with overweight only at one age or at both ages had a significantly increased risk of PCOS (online suppl. Table S2).

Childhood height was linearly and positively associated with the risk of PCOS and the associations were similar at all childhood ages (Table 5). In the analyses on growth in height in girls with body size information at the age of 7 and 13 years, we found that girls who were tall during childhood or changed from taller than average to average height had an increased risk of PCOS compared with those with average height growth (Table 5). In contrast, girls who grew taller than average did not have an increased risk of PCOS.

\section{Discussion}

In this large-scale, population-based, cohort study of Danish girls, we found that size at birth was not related to a subsequent risk of PCOS. At childhood ages, above-average BMI values or tall stature was associated with a significantly increased risk of PCOS. Furthermore, we found that, compared with girls with overweight at the age of 7 or 13 years, girls without overweight at either 7 or 13 years, but especially those without overweight at both of these ages, had a lower risk of PCOS. Finally, persistently tall girls or girls who changed from tall to average height had a higher risk of PCOS than those with average height growth.

We did not find associations between birthweight and PCOS risk. Despite speculations of such an association, findings from previous studies are inconsistent. A recent meta-analysis of 14 studies found that a low birthweight might increase the risk of PCOS [9], but a subsequent, largescale, registry-based study from Sweden did not find an association [24]. Thus, based on the current evidence, the role of birthweight in the etiology of PCOS is still uncertain. 


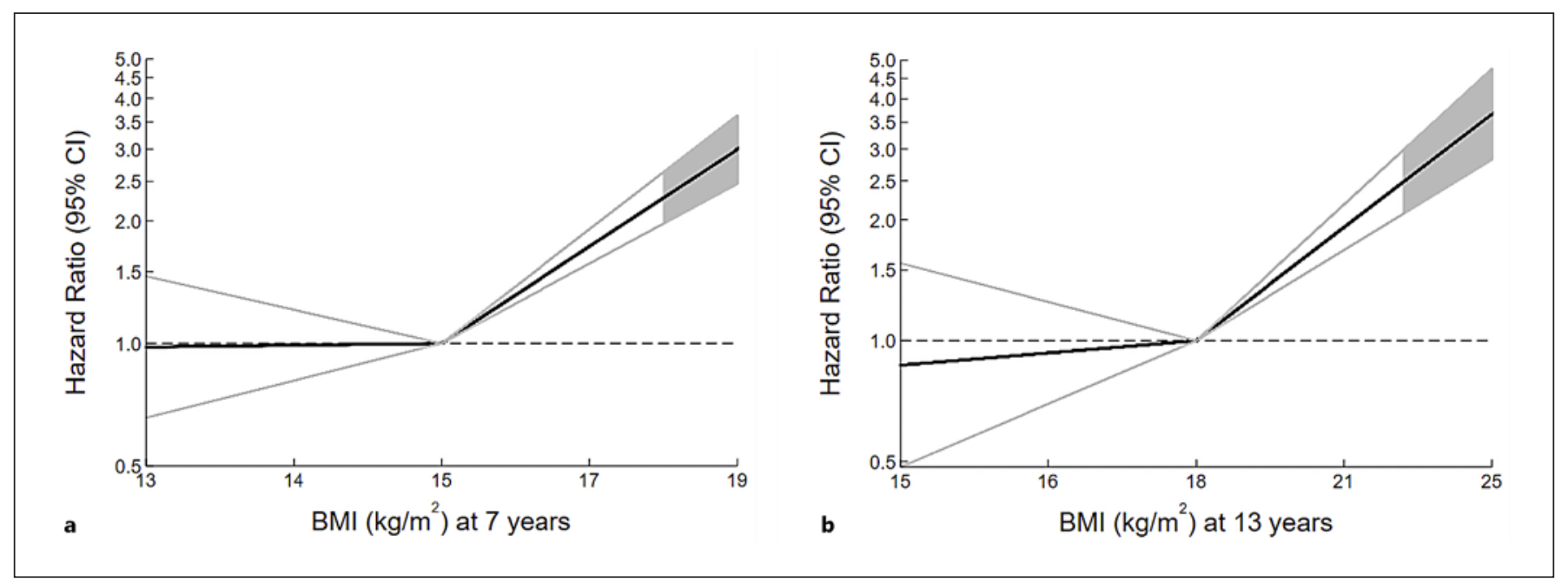

Fig. 1. Associations between childhood BMI $Z$ scores at the age of 7 (a) and 13 (b) years and the risk of developing polycystic ovary syndrome. The associations are stratified by birth cohort. The associations were modeled using linear splines with a knot at a $Z$ score of 0 . The $x$ axis is labeled with the corresponding BMI values. The grey area indicates the hazard ratios for BMI values of overweight as defined by the International Obesity Task Force (IOTF) criteria.

Table 3. Associations between childhood weight status at each age, from 7 to 13 years, and the risk of developing polycystic ovary syndrome ${ }^{1}$

\begin{tabular}{llrlll}
\hline Age, years & Weight status & \multicolumn{1}{l}{$N$} & $n$, cases & HR & 95\% CI \\
\hline 7 & non-overweight & 53,042 & 386 & 1.00 (ref.) & \\
& overweight & 6,836 & 152 & 2.83 & $2.34-3.42$ \\
\hline 8 & non-overweight & 51,555 & 378 & 1.00 (ref.) & \\
& overweight & 6,920 & 153 & 2.73 & $2.26-3.30$ \\
\hline 9 & non-overweight & 44,595 & 298 & 1.00 (ref.) & \\
& overweight & 6,485 & 142 & 2.85 & $2.32-3.50$ \\
\hline 10 & non-overweight & 40,559 & 268 & 1.00 (ref.) & \\
& overweight & 6,028 & 129 & 2.72 & $2.19-3.37$ \\
\hline 12 & non-overweight & 39,991 & 245 & 1.00 (ref.) & \\
& overweight & 5,677 & 121 & 2.94 & $2.35-3.67$ \\
\hline 13 & non-overweight & 39,397 & 228 & 1.00 (ref.) & \\
& overweight & 5,624 & 131 & 3.39 & $2.72-4.22$ \\
\hline
\end{tabular}

$\mathrm{HR}$, hazard ratio; CI, confidence interval.

${ }^{1}$ Stratified by birth cohort.

We found that above-average BMI (e.g., 15.3 in a 7-year-old girl) was associated with a significantly higher risk of PCOS. Notably, these associations arise at levels way below those considered as overweight by international contemporary cut-off values. When childhood BMI was classified as overweight or non-overweight, overweight was associated with a nearly 3 -fold higher risk of PCOS than non-overweight. These findings illustrate that BMI at childhood ages before PCOS is typically diagnosed is relevant for this disorder. Our study is based on measured anthropometric values and registered diagnoses; this contrasts with most other studies in this field which have relied on self-reported childhood size and only few cases of self-reported PCOS or PCOS-related symptoms. None- 
Table 4. Associations between different patterns of change in weight status from the ages 7 to 13 years and the risk of polycystic ovary syndrome, with girls with overweight at both ages as the reference ${ }^{1}$

\begin{tabular}{lrrrr}
\hline Weight status $^{2}$ & $N$ & Cases, $n$ & HR & $95 \%$ CI \\
\hline Non-overweight at 7 and at 13 years & 32,175 & 177 & 0.26 & $0.19-0.34$ \\
Non-overweight at 7 years and overweight at 13 years & 2,063 & 34 & 0.69 & $0.42-0.96$ \\
Overweight at 7 years and non-overweight at 13 years & 1,539 & 19 & 0.53 & $0.32-0.88$ \\
Overweight at 7 and at 13 years & 2,508 & 65 & 1.00 (ref.) \\
\hline
\end{tabular}

$\mathrm{HR}$, hazard ratio; CI, confidence interval.

${ }^{1}$ Stratified by birth cohort.

${ }^{2}$ Overweight as defined by the International Obesity Task Force criteria.

Table 5. Associations between childhood height at each age from 7 to 13 years and different hypothetical patterns of growth in height from age 7 to 13 years, and the risk of polycystic ovary syndrome (per $Z$ score) $)^{1}$

\begin{tabular}{lllll}
\hline Age, years & $N$ & Cases, $n$ & HR & $95 \%$ CI \\
\hline 7 & 59,878 & 538 & 1.19 & $1.09-1.29$ \\
8 & 58,475 & 531 & 1.21 & $1.11-1.32$ \\
9 & 51,080 & 440 & 1.22 & $1.11-1.34$ \\
10 & 46,587 & 397 & 1.28 & $1.16-1.41$ \\
11 & 45,668 & 366 & 1.23 & $1.11-1.36$ \\
11 & 45,021 & 359 & 1.21 & $1.09-1.34$ \\
13 & 42,340 & 339 & 1.12 & $1.01-1.25$ \\
\hline
\end{tabular}

\section{Height growth pattern ${ }^{2}$}

Average growth

Persistently taller than average ${ }^{4}$

Growing taller than average ${ }^{5}$

Changing from taller than average to average ${ }^{6}$

$\mathrm{HR}$, hazard ratio; $\mathrm{CI}$, confidence interval.

${ }^{1}$ Stratified by birth cohort.

${ }^{2}$ Of 38,285 women, 295 had a PCOS diagnosis.

${ }^{3} \mathrm{~A} Z$ score of zero at both ages, i.e., 7 and 13 years.

${ }^{4} \mathrm{~A} Z$ score of 0.5 at both ages, i.e., 7 and 13 years.

${ }^{5} \mathrm{~A} Z$ score of zero at age 7 years and 0.5 at 13 years.

${ }^{6} \mathrm{~A} Z$ score of 0.5 at age 7 years and zero at 13 years.

theless, consistent with our study, 5 other studies found that a high BMI or a large body size in girls was associated with an increased risk of self-reported hirsutism, menstrual problems, or PCOS, compared with girls with a normal weight or a smaller body size $[10-13,15]$.

Furthermore, we showed that girls without overweight during childhood, or with overweight in early or late childhood only, had a lower risk of PCOS than those with overweight at both ages. These findings indicate that the longer the exposure to the harmful effects of childhood adiposity, the higher the subsequent risk of PCOS. Generally consistent with our findings, a small case-control study examined childhood BMI trajectories from birth to the age of 13 years and found that the BMI level was higher in women with PCOS than in controls [14]. We found that if a girl remitted from overweight during childhood, she decreased her risk of PCOS. Although the risk remained higher than in girls without overweight at either age, these findings highlight the importance of weight management in childhood.

Childhood height was positively and strongly associated with the risk of PCOS. To put our findings into perspective, compared with an average-height 7-year-old girl, one who was approximately $5.2 \mathrm{~cm}$ taller (equivalent to a $\mathrm{Z}$ score of 1) has a $20 \%$ increased risk of PCOS. In addition, girls who were tall throughout childhood or changed from average to below-average height had a higher PCOS risk than those with average height growth (equivalent to approx. $34 \mathrm{~cm}$ between the ages of 7 and 13 years). We speculate that the growth pattern associated with a relative decrease in height reflects girls who had an early growth spurt but did not yet have their pubertal growth spurt. Interestingly, girls who grew taller than average during childhood did not have an increased risk of developing PCOS. We did not find any studies in the literature supporting these observations on childhood height and linear growth and PCOS risk. The associations we found are in the opposite direction to those seen for cardiometabolic diseases $[16,25,26]$, suggesting that other pathways may be underlying the associations with PCOS risk. Our novel findings suggest that childhood height is a marker of the risk of developing PCOS.

It is biologically plausible that excess weight in childhood contributes to PCOS development as well as exacerbating the severity of the syndrome through a worsen- 
ing of metabolic and reproductive disturbances. Excess adiposity is associated with increased insulin resistance, and compensatory hyperinsulinemia stimulates the ovarian production of androgens and inhibits the production of sex-hormone-binding globulin. Thus, the increased concentration of free androgens may promote PCOS pathogenesis $[8,27]$. Furthermore, insulin resistance may contribute directly to ovarian dysfunction [28]. We cannot entirely disentangle whether tracking of child BMI into adult ages [29] may partially have affected our findings, but, considering that PCOS emerges early in life, it is unlikely that tracking entirely explains our findings. In our study, height indicated a risk of PCOS, but it is unlikely to be an underlying causal factor for this disorder. It is plausible that pleiotropic genetic effects link height and PCOS, but this is purely speculative. Thus, early childhood may represent a critical time window during which body size influences PCOS development.

The strengths of this study include the prospective study design that included a large population of virtually all Copenhagen schoolgirls. Weights and heights were measured, thus avoiding recall bias. Compared to most other studies that used self-reported information on a diagnosis of PCOS, with only 1 or 2 symptoms or even just hirsutism, we included women with PCOS diagnosed by a clinician. Furthermore, follow-up extended over many years with a minimal loss of individuals in a nation that provides universal health care; this minimized any potential selection bias in the cohort.

Our study also has limitations. During the study period, the diagnostic criteria for PCOS changed, most notably with the introduction of the Rotterdam criteria in 2003. We speculate that any impact of changes in diagnostic criteria on the associations between BMI and height, respectively, and PCOS risk across time was minimal; in support of this, $>90 \%$ of our cases were diagnosed after the early 2000s. Furthermore, although numerous medical and socioeconomic changes occurred during the study period, associations between early-life body size and PCOS development did not differ by birth cohort. Our study included only women with PCOS who underwent hospital-based investigations and not any women treated exclusively in primary care. The prevalence of PCOS in our study was thus low and likely included the most severe cases. This may have affected our results and limited the generalizability of our findings to women with more severe phenotypes of PCOS. Further prospective studies are needed to confirm if childhood body size is related to PCOS risk and whether these associations differ according to the PCOS phenotype.

Early-Life Body Size and PCOS
Information on adult obesity and body composition was not available in our study. However, although adult obesity and PCOS are closely interrelated, their temporal relation is complex and not fully understood. By including only early-life exposures, we enhanced temporality in our study and minimized the potential for reverse causality. In addition, information on potential explanatory factors such as pubertal timing and a family history of PCOS or genetics was not available. The girls in the cohort were mainly of Caucasian descent, so the findings are likely generalizable to contemporary populations of girls in many Western countries.

In conclusion, we found that birthweight is not related to PCOS risk, but that above-average BMI and taller height are associated with an increased risk of PCOS, thus suggesting that risks can be identified based on childhood body size. Increased risks of PCOS appeared at BMI values that were significantly lower than those defined as overweight by international contemporary criteria. With the worldwide progression of the childhood obesity epidemic, the prevalence of PCOS will likely increase, causing a significant health burden for both women and society. Our findings emphasize the importance of attaining and maintaining a healthy weight during childhood.

\section{Acknowledgement}

The CSHRR was initiated and planned by Dr. Thorkild I.A. Sørensen and built by the Institute of Preventive Medicine, The Capital Region of Denmark.

\section{Statement of Ethics}

This study was approved by the Danish Data Protection Agency (Datatilsynet), approval No. 2012-58-0004. According to Danish law, ethics approval and informed consent are not required for registry-based studies.

\section{Conflict of Interest Statement}

Dr. Jens-Christian Holm reports obesity related services to patients and health care professionals. All other authors have no declarations.

\section{Funding Sources}

The work presented in this article is supported in part by the Novo Nordisk Foundation grant NNF17OC0028338 and the Independent Research Fund Denmark, Medical Science, grant DFF- 
7016-00390. The sponsor had no role in the study design; collection, analysis, and interpretation of data; writing of the report; or the decision to submit the report for publication.

\section{Author Contributions}

J.A. and J.L.B. conceived and designed the original study with substantial input from L.G.B., J.L.B. contributed data, J.A. and L.G.B. conducted the statistical analyses, J.A. and J.L.B. wrote the manuscript and developed the tables and figures, and all authors critically reviewed the manuscript and approved the final version.

\section{Data Availability}

The data used in this study are based on a combination of data with personal identification numbers from "third parties", the Copenhagen School Health Records Register, hosted by the Center for Clinical Research and Prevention, and data from national health registries. According to Danish law, this information cannot be publicly available. Access to the subset of data included in this study can be gained through submitting a project application to the corresponding author, J.L.B., and pending approval by the data steering committee.

\section{References}

1 Dumesic DA, Oberfield SE, Stener-Victorin E, Marshall JC, Laven JS, Legro RS. Scientific Statement on the Diagnostic Criteria, Epidemiology, Pathophysiology, and Molecular Genetics of Polycystic Ovary Syndrome. Endocr Rev. 2015 Oct;36(5):487-525.

2 Azziz R, Carmina E, Chen Z, Dunaif A, Laven JS, Legro RS, et al. Polycystic ovary syndrome. Nat Rev Dis Primers. 2016 Aug;2(1):16057.

3 Bozdag G, Mumusoglu S, Zengin D, Karabulut E, Yildiz BO. The prevalence and phenotypic features of polycystic ovary syndrome: a systematic review and meta-analysis. Hum Reprod. 2016 Dec;31(12):2841-55.

4 Gottschau M, Kjaer SK, Jensen A, Munk C, Mellemkjaer L. Risk of cancer among women with polycystic ovary syndrome: a Danish cohort study. Gynecol Oncol. 2015 Jan;136(1): 99-103.

5 Rubin KH, Glintborg D, Nybo M, Abrahamsen B, Andersen M. Development and Risk Factors of Type 2 Diabetes in a Nationwide Population of Women With Polycystic Ovary Syndrome. J Clin Endocrinol Metab. 2017 Oct;102(10):3848-57.

6 Glintborg D, Rubin KH, Nybo M, Abrahamsen B, Andersen M. Cardiovascular disease in a nationwide population of Danish women with polycystic ovary syndrome. Cardiovasc Diabetol. 2018 Mar;17(1):37.

7 Hoeger KM. Obesity and lifestyle management in polycystic ovary syndrome. Clin Obstet Gynecol. 2007 Mar;50(1):277-94.

8 Lim SS, Davies MJ, Norman RJ, Moran LJ. Overweight, obesity and central obesity in women with polycystic ovary syndrome: a systematic review and meta-analysis. Hum Reprod Update. 2012 Nov-Dec;18(6):618-37.

9 Sadrzadeh S, Hui EV, Schoonmade LJ, Painter RC, Lambalk CB. Birthweight and PCOS: systematic review and meta-analysis. Hum Reprod Open. 2017 Aug;2017(2):hox010.

10 Lake JK, Power C, Cole TJ. Women's reproductive health: the role of body mass index in early and adult life. Int J Obes Relat Metab Disord. 1997 Jun;21(6):432-8.
11 Laitinen J, Taponen S, Martikainen H, Pouta A, Millwood I, Hartikainen AL, et al. Body size from birth to adulthood as a predictor of self-reported polycystic ovary syndrome symptoms. Int J Obes Relat Metab Disord. 2003 Jun;27(6):710-5.

12 Ollila MM, Piltonen T, Puukka K, Ruokonen A, Järvelin MR, Tapanainen JS, et al. Weight Gain and Dyslipidemia in Early Adulthood Associate With Polycystic Ovary Syndrome: Prospective Cohort Study. J Clin Endocrinol Metab. 2016 Feb;101(2):739-47.

13 Li J, Eriksson M, He W, Hall P, Czene K. Associations between childhood body size and seventeen adverse outcomes: analysis of 65,057 European women. Sci Rep. 2017 Dec; 7(1):16917.

14 Koivuaho E, Laru J, Ojaniemi M, Puukka K, Kettunen J, Tapanainen JS, et al. Age at adiposity rebound in childhood is associated with PCOS diagnosis and obesity in adulthood-longitudinal analysis of BMI data from birth to age 46 in cases of PCOS. Int J Obes. 2019 Jul;43(7):1370-9.

15 He Y, Tian J, Blizzard L, Oddy WH, Dwyer T, Bazzano LA, et al. Associations of childhood adiposity with menstrual irregularity and polycystic ovary syndrome in adulthood: the Childhood Determinants of Adult Health Study and the Bogalusa Heart Study. Hum Reprod. 2020 May;35(5):1185-98.

16 Stefan N, Häring HU, Hu FB, Schulze MB. Divergent associations of height with cardiometabolic disease and cancer: epidemiology, pathophysiology, and global implications. Lancet Diabetes Endocrinol. 2016 May;4(5): 457-67.

17 Baker JL, Olsen LW, Andersen I, Pearson S, Hansen B, Sørensen TI. Cohort profile: the Copenhagen School Health Records Register. Int J Epidemiol. 2009 Jun;38(3):656-62.

18 Jensen CB, Gamborg M, Heitmann B, Sørensen TI, Baker JL. Comparison of birth weight between school health records and medical birth records in Denmark: determinants of discrepancies. BMJ Open. 2015 Nov; 5(11):e008628.
19 Pedersen CB. The Danish Civil Registration System. Scand J Public Health. 2011 Jul;39(7 Suppl):22-5.

20 Rotterdam EA; Rotterdam ESHRE/ASRMSponsored PCOS Consensus Workshop Group. Revised 2003 consensus on diagnostic criteria and long-term health risks related to polycystic ovary syndrome. Fertil Steril. 2004 Jan;81(1):19-25.

21 Cole TJ, Green PJ. Smoothing reference centile curves: the LMS method and penalized likelihood. Stat Med. 1992 Jul;11(10):130519.

22 Cole TJ, Lobstein T. Extended international (IOTF) body mass index cut-offs for thinness, overweight and obesity. Pediatr Obes. 2012 Aug;7(4):284-94.

23 Baker JL, Olsen LW, Sørensen TIA. Weight at birth and all-cause mortality in adulthood. Epidemiology. 2008 Mar;19(2):197-203.

24 Valgeirsdottir H, Vanky E, Sundström-Poromaa I, Roos N, Løvvik TS, Stephansson O, et al. Prenatal exposures and birth indices, and subsequent risk of polycystic ovary syndrome: a national registry-based cohort study. BJOG. 2019 Jan; 126(2):244-51.

25 Silventoinen K, Baker JL, Sørensen TI Growth in height in childhood and risk of coronary heart disease in adult men and women. PLoS One. 2012;7(1):e30476.

26 Bjerregaard LG, Jensen BW, Baker JL. Height at Ages 7-13 Years in Relation to Developing Type 2 Diabetes Throughout Adult Life. Paediatr Perinat Epidemiol. 2017 Jul;31(4):284-92.

27 Anderson AD, Solorzano CM, McCartney CR. Childhood obesity and its impact on the development of adolescent PCOS. Semin Reprod Med. 2014 May;32(3):202-13.

28 Thong EP, Codner E, Laven JS, Teede H. Diabetes: a metabolic and reproductive disorder in women. Lancet Diabetes Endocrinol. 2020 Feb;8(2):134-49.

29 Aarestrup J, Bjerregaard LG, Gamborg M, Ängquist L, Tjønneland A, Overvad K, et al. Tracking of body mass index from 7 to 69 years of age. Int J Obes. 2016 Sep;40(9):137683. 